\title{
BMJ Open Physicians' role in the development of inappropriate polypharmacy among older adults in Iran: a qualitative study
}

\author{
Seyede Salehe Mortazavi, ${ }^{1}$ Mohsen Shati, ${ }^{2}$ Seyed Kazem Malakouti, ${ }^{1}$ \\ Hamid Reza Khankeh, ${ }^{3}$ Shiva Mehravaran, ${ }^{4}$ Fazlollah Ahmadi $^{5}$
}

To cite: Mortazavi SS, Shati M, Malakouti SK, et al. Physicians' role in the development of inappropriate polypharmacy among older adults in Iran: a qualitative study. BMJ Open 2019;9:e024128. doi:10.1136/ bmjopen-2018-024128

- Prepublication history for this paper is available online. To view these files, please visit the journal online (http://dx.doi org/10.1136/bmjopen-2018024128).

Received 12 May 2018 Revised 26 February 2019 Accepted 19 March 2019

Check for updates

(C) Author(s) (or their employer(s)) 2019. Re-use permitted under CC BY-NC. No commercial re-use. See rights and permissions. Published by BMJ.

${ }^{1}$ School of Behavioral Sciences and Mental Health, Iran University of Medical Sciences, Tehran, Iran

${ }^{2}$ Mental Health Research Center, School of Behavioral Sciences and Mental Health, Iran University of Medical Sciences, Tehran, Iran

${ }^{3}$ Department of Nursing, University of Social Welfare and Rehabilitation Sciences (USWR), Tehran, Iran

${ }^{4}$ Department of Ophthalmology, Stein Eye Institute, David Geffen School of Medicine, University of California, Los Angeles, USA

${ }^{5}$ Department of Nursing, Tarbiat Modares University, Tehran, Iran

Correspondence to

Dr Mohsen Shati;

shati.m@iums.ac.ir

\section{ABSTRACT}

Objectives The use of unnecessary or excessive medications (inappropriate polypharmacy) is a major health challenge among older adults which is driven by several factors. This study aims to provide in-depth descriptions of the physician's role in the development of inappropriate polypharmacy among older adults in Iran. Design Qualitative content analysis of interviews, field notes and other relevant documents available (eg, medical records). Data collection and analyses were done concurrently to guide the sampling process.

Setting Three purposively selected referral hospitals in Tehran, Iran.

Participants A total of 7 physicians, 10 older adults, 3 caregivers and 3 pharmacists with a median age of 54 (IQR 23) years were recruited through convenience sampling.

Results Emerged categories included misdiagnosis, inappropriate prescribing, insufficient patient education, poor communication, unprofessional behaviour and limited perspectives which highlight the role of physicians in the development of inappropriate polypharmacy among older adults in Iran under the main concept of poor medical practice.

Conclusion This study provides valuable insight on the role of physicians in the development of inappropriate polypharmacy among the elderly in the healthcare setting in Iran by exploring the viewpoints of physicians, patients, caregivers and pharmacists. Physicians can be an influential factor in tackling this challenge through proper diagnosis, prescription, patient education and follow-up. In Iran, physicians' practice styles are affected by potentially adverse factors such as the novelty of geriatric medicine, lack of a referral system, patient unfamiliarity with the system and lack of a monitoring system for multiple prescriptions. Furthermore, clinics tend to be overcrowded and visit fees can be low; in this setting, lack of physician assistants leads to limited time allocation to each patient and physician dissatisfaction with their income.

\section{INTRODUCTION}

Currently, available medications in the global market are abundantly diverse and are the first line of treatment for many diseases. Although medication use appears inevitable in the treatment of many diseases, their various side effects cannot be overlooked.

\section{Strengths and limitations of this study}

- This is the first study to explore the role of physicians in the development of inappropriate polypharmacy in the context of the Iranian health system.

- We triangulated data sources and participants to ensure robustness of results.

- Engagement with the data was prolonged (about 1 year) to ensure credibility and acceptability of results.

- This inductive content analysis was conducted in Tehran, the capital city of Iran. Although this may be considered as a limitation in transferability, results could be transferable to other countries with similar healthcare systems.

Hence, issues related to medication use are one of the challenges in healthcare. ${ }^{12}$

Unnecessary and excessive use of medications has long been a matter of interest to researchers, healthcare providers and health decision-makers. Not only does the problem impose cost and burden to the health system, ${ }^{3}$ it is also responsible for various adverse events including increased risk of adverse reactions and interactions, ${ }^{4}$ reduced adherence to therapy, ${ }^{5}$ increased frequency of care visits and hospitalisation, and increased risk of patient morbidity and mortality. ${ }^{67}$

Polypharmacy refers to the use of unnecessary or more than medically necessary medications. ${ }^{45}$ Although there is no consensus on the definition of polypharmacy, some define it based on the number of medications a person may be taking. ${ }^{8}$ Regardless of the definition, current literature confirms the association of polypharmacy with the above-mentioned consequences. Since the concurrent use of multiple medications does not necessarily denote incorrect use or prescription of medications, polypharmacy can be classified as appropriate and inappropriate. In appropriate polypharmacy, the patient can take a combination of medications, which are 
clinically indicated on account of patient condition and clinical evidence. Inappropriate polypharmacy, on the other hand, occurs when patients use or are prescribed more medicines than are clinically indicated ${ }^{5}$ which is the definition used in this study.

One of the potential causes of inappropriate polypharmacy is inappropriate prescribing which refers to misprescribing, overprescribing and underprescribing. Misprescribing involves an incorrect dose, frequency, administration route or duration of treatment, or the use of medications that are likely to cause clinically significant drug-drug or drug-disease interactions while low-risk alternatives exist. Overprescribing involves prescribing medications for which no clear clinical indication exists. Both misprescribing and overprescribing are common causes of inappropriate polypharmacy. ${ }^{9}$

In screening for inappropriate polypharmacy, multiple tools have been proposed and used in various studies ${ }^{1011}$; these include the Medication Appropriateness Index (MAI) ${ }^{12}$ Beers criteria for determining potentially inappropriate medication (PIM),${ }^{13}$ the screening tool of older person's prescriptions (STOPP) and the screening tool to alert doctors to the right treatment (START) ${ }^{14}$ STOPP is composed of 80 clinically significant criteria for potentially inappropriate prescribing in older people. Each criterion is accompanied by a concise explanation as to why the prescribing practice is potentially inappropriate. START consists of 34 evidence-based prescribing indicators for commonly encountered diseases in older people. $^{14}$

Inappropriate polypharmacy may occur at any age, but given the unique circumstances of older adults, it is more common in this age group. ${ }^{58}$ In addition to higher prevalence of chronic diseases, ${ }^{35}$ the aged population is at risk of multimorbidities. ${ }^{5}$ To treat these multiple concurrent diseases, older patients often use multiple medications. On the other hand, due to age-related physiological changes in the aged population, the impact of medications can vary, and responses to medication may be different and less predictable than other age groups. ${ }^{15}$ Therefore, medication-related problems among older adults are much more serious and should be addressed more vigilantly. ${ }^{6}$ For instance, the definition of excess medications and quantifying the threshold for the sufficient amount of medications is different for the aged population, and the duration of the prescription should be properly adjusted as well. Other factors that can contribute to the development of inappropriate polypharmacy among older adults include use of PIM ${ }^{16}$ self-medication, ${ }^{5}$ multiple therapeutic regimens and prescriptions by multiple physicians. ${ }^{6}$ Therefore, although patients, physicians and pharmacists are all likely to have key roles in its development and prevention, ${ }^{17}$ physicians' prescriptions are the main contributing factor (except in self-medication), and thus, physicians' role deserves a closer examination.

In Iran, the population of older adults is growing. ${ }^{18}$ Studies have shown that chronic diseases ${ }^{19} 20$ and polypharmacy $^{2122}$ are quite common in this population. In addition, the healthcare system in Iran and the patterns of care and service delivery have unique features compared with other countries. ${ }^{23}$ For example, patient referral in the primary healthcare system, which is functional in rural areas, does not exist in urban areas except in two pilot provinces (Fars and Mazandaran). In addition, lack of an integrated health information system creates challenges in connecting system components, evaluating the quality of services and examining the efficiency of the health system. ${ }^{23}{ }^{24}$ In light of the above discussion, this study was designed and conducted to answer the question as to what role physicians have in the development of inappropriate polypharmacy among elderly Iranians.

\section{METHODS}

\section{Study design}

This study is a part of a larger qualitative study which was conducted with the objective of examining medication-related problems in Iranian elderly adults. Another part of this study that focuses on self-medication among elderly Iranians has been published elsewhere. ${ }^{25}$ We applied the qualitative content analysis approach described by Graneheim and Lundman. ${ }^{26}$

\section{Participants and study setting}

The research was based in Tehran, the capital of Iran. In the first step, three major referral hospitals (Rasoul Akram, Imam Khomeini and Hasheminejad) were purposively selected. Then, within each setting, convenience sampling was used to select individuals who had some form of living experience with the studied phenomenon and appeared capable of providing rich data to the investigator. Study participants consisted of four groups: elderly adults with inappropriate polypharmacy, caregivers, pharmacists and physicians. Any individual who was unwilling to participate in the study (no consent) or complete the interview (withdrawal) as well as those with poor cooperation or communication with the interviewer for any reason, such as cognitive deficits or psychological disorders, were excluded. These criteria were assessed by the team physician, and exclusions were done at his discretion.

To define 'elderly adults', we used the definition proposed by WHO, which suggests a cut-off age of 60 years for low/middle-income countries. ${ }^{27}$ According to the 2011 Population and Housing Census in Iran, 9.2\% of the population of Tehran County were adults over 60 years of age. ${ }^{18}$ The first inclusion criterion for the older participants of this study was the simultaneous use of at least four medications in the past 6 months. ${ }^{5}$ For these cases, all medications taken routinely were reviewed and counted. Then, the team physician reviewed their medical history and medications profile. Eventually, older adults who had at least one excessive or unnecessary medication based on the MAI were selected.

The inclusion criteria for caregivers were living with the elderly and/or having comprehensive information about 
the treatment regimen of the elderly person in their care. Selected physicians were those whose patients were mostly older adults.

To reach a complete understanding of different aspects of the phenomena, a number of factors were taken into consideration during the selection process. These included maximum variation in terms of age, gender, socioeconomic level, duration of medication consumption and comorbid chronic conditions for selecting elderly individuals. For example, to achieve maximum variation in terms of multimorbidity, the type of disease was taken into consideration in the selection of elderly participants to include a combination of diseases common among older adults. These included endocrine and metabolic disorders (eg, diabetes, thyroid disorders, lipid disorders), cardiovascular diseases (eg, hypertension, ischaemic heart disease), musculoskeletal disorders (eg, arthritis, rheumatic diseases), malignancies, digestive disorders, respiratory diseases, disorders of the genitourinary system and neurological disorders. For physicians, the type of specialty and years of experience, and for pharmacists, the socioeconomic status of the location of the pharmacy, years of experience and employment sector (public or private) were taken into consideration.

\section{Patient and public involvement}

Patients and the public were not involved in the design, recruitment or conduct of this study.

\section{Data collection and analysis}

Data for this study were collected from October 2016 to September 2017 using semistructured face-to-face interviews, field notes and available documents such as medications available at home, medical records and proposals approved by the Council of Ministers regarding health services. Data collection from informants was done through semistructured interviews. All interviews were done in Persian and conducted by the first author (SSM) who is a trained interviewer and has years of experience in providing primary care to older adults and research on issues related to their problems. Interviews were conducted at a location approved by the participants. Interview locations for elderly individuals and their caregivers were their residence, a clinic or a hospital. Physicians and pharmacists chose to have their interviews at their workplaces.

During the interview sessions, only the research physician (who was introduced as a health researcher) and the interviewer were present to ensure the participants of their privacy. Study participants were able to interact and spoke comfortably about their experiences. All interviews were recorded with a digital recorder, and a topic guide was used to guide the interviews. A separate topic guide was prepared for each group of participants based on a review of the literature, and these were fine-tuned by consulting all research team members. The guides were then reviewed and revised after pilot interviews with one geriatric medicine specialist, one elderly individual, one caregiver and one pharmacist. Data from these interviews were not included in this study. This review process was applied after each interview and analysis, and revisions continued throughout the study.

Interviews with older adults began with the open-ended question 'Think of an instance when you went to a doctor, and you remember the details well' followed with questions such as 'Can you elaborate?', 'Could you clarify what you mean?', 'Do you have any particular experience in this regard?'. These questions were then followed by probing/prompt questions to explore concepts in terms of their properties and dimensions. At the conclusion, the participants were asked if there was anything they wished to add. A second interview was scheduled if the interview was not complete in the first session.

Data analysis was performed using an inductive approach. In this approach, the concepts are derived from the data and not previous knowledge. ${ }^{28}$ Qualitative data analysis software (MAXQDA V.10) was used to facilitate data analysis. All recordings were transcribed verbatim within 3 days after the interview, and then thoroughly reviewed several times. This approach allowed the researcher to get a general sense of the interview. During the next rounds of review, words and sentences related to the research question were identified as meaning units. These meaning units were coded based on their hidden contents. Codes with similar contents were classified into more abstract subcategories and labelled accordingly. Finally, study categories were determined by comparing subcategories with each other and reflecting on their latent content. ${ }^{26}$

Results of the analysis of each interview directed the next interview, and sampling continued until data saturation, that is, when concept categories were complete, and the researcher believed additional data collection would not provide a more in-depth understanding of the phenomenon. Data saturation was achieved for the entire sample and not per different subgroups.

\section{Ethical considerations}

All potential participants were provided with the necessary information regarding the goals and processes of the study, and only those who signed informed consents were enrolled in the study. Participants were ensured that their information would be treated in confidence, their anonymity would be protected and that the information collected in the study would be used only for research purposes. They were also ensured that their identities would remain anonymous in publications. Participants were informed that they were free to leave the study at any stage of the study. Voice recording during the interviews was done with their permission and audio files were deleted after transcription. During the study, all data were stripped of subject identifiers, and the codes linking data to the identifiers were stored in a secure location. At the conclusion of the study, all data were transferred to the databank maintained by the Mental Health Research Centre, Iran University of Medical Sciences, where it is 
only accessible by the principal investigator and first author.

\section{Trustworthiness}

To ensure scientific accuracy and robustness of data, different methods practised in qualitative research were used. ${ }^{29}$ In some cases, the researcher asked the participants to review the coded transcripts to verify the accuracy and appropriateness of the extracted code compared with their personal experiences and opinions, and corrections were made accordingly.

A portion of the coded data was reviewed by three content experts to examine the data analysis and judge the validity. In addition, all interviews were coded by two independent individuals, and discrepancies were discussed at research meetings to make final decisions. To confirm the accuracy of the findings and ensure they are supported by the collected data, a third researcher (who has extensive experience in qualitative research and was not directly involved with our data collection or analysis) monitored the data collection and analysis processes and reviewed the results.

In addition, maximum variation sampling provided a wider range of experiences that would enhance the credibility of the data. Furthermore, to allow transferability, a clear and complete description of the study is provided, so that the reader can extract the necessary information about the research question, the methodology, sample selection, study setting, data collection, data analysis, results, challenges and limitations of the study.

\section{Reporting}

This study is reported in accordance with the Consolidated Criteria for Reporting Qualitative Research guidelines. ${ }^{30}$

\section{RESULTS}

There were 23 participants in this study; 14 females and 9 males. Of these, 10 were older adults, 3 were their caregivers, 7 were physicians and 3 were pharmacists. The mean age of the participants was $56.69 \pm 13.65$ years, and the mean age of the elderly group was $69.60 \pm 8.42$ years. Among older adult participants, two were illiterate, six had some grade school education and two had a high school diploma or higher. The number of medications (prescribed and non-prescribed) used by these patients ranged from a minimum of 5 to a maximum of 17 per day, and the number of concurrent diseases ranged between 2 and 5. The physicians' areas of expertise included general medicine, geriatrics, internal medicine, psychiatry, pain fellowship and traditional medicine. Participants' characteristics are presented in tables 1 and 2. A total of 26 interview sessions were held. The length of the interviews ranged between 60 and $90 \mathrm{~min}$.

The main theme that emerged from the data was poor medical practice. This was based on six categories of misdiagnosis, inappropriate prescribing, insufficient patient education, poor communication, unprofessional

\begin{tabular}{lc}
$\begin{array}{l}\text { Table } 1 \\
\text { study }\end{array}$ & Characteristics of older adults participating in this \\
\hline Variables & $\mathbf{N}(\%)$ \\
\hline $\begin{array}{l}\text { Gender } \\
\text { Male }\end{array}$ & $2(20)$ \\
\hline female & $8(80)$ \\
Age & \\
$60-64$ & $4(40)$ \\
$65-69$ & $2(20)$ \\
$70-74$ & $2(20)$ \\
$75-79$ & $0(0)$ \\
$>80$ & $2(20)$ \\
Marital status & $8(80)$ \\
\hline Married & $2(20)$ \\
Widowed & $10.8(3.4)$ \\
No of medications used & \\
Mean (SD) &
\end{tabular}

behaviour and limited perspectives, which were derived from 20 subcategories (table 3 ). These are discussed in the following sections.

\section{Misdiagnosis}

Subcategories forming the 'misdiagnosis' category were 'insufficient knowledge about symptoms in older patients', 'skipping physical examinations' and 'incomplete history taking'. Misdiagnosis by a physician can not only cause a misprescription, and consequently,

Table 2 Characteristics of caregivers, physicians and pharmacists participating in this study

\begin{tabular}{lc}
\hline Variables & $\mathbf{N}(\%)$ \\
\hline $\begin{array}{l}\text { Gender } \\
\text { male }\end{array}$ & $7(54)$ \\
female & $6(46)$ \\
\hline Age & \\
$<45$ & $5(38)$ \\
$45-49$ & $4(31)$ \\
$50-54$ & $3(23)$ \\
$55-59$ & $0(0)$ \\
$>60$ & $1(8)$ \\
\hline Marital status & \\
\hline Married & $11(85)$ \\
\hline Single & $1(7.5)$ \\
\hline Divorced & $1(7.5)$ \\
Role & \\
\hline Physician & $7(54)$ \\
\hline Pharmacist & $3(23)$ \\
\hline Caregiver & $3(23)$ \\
\hline
\end{tabular}


Table 3 Physicians' role in the development of inappropriate polypharmacy among older adults based on the participants' experiences

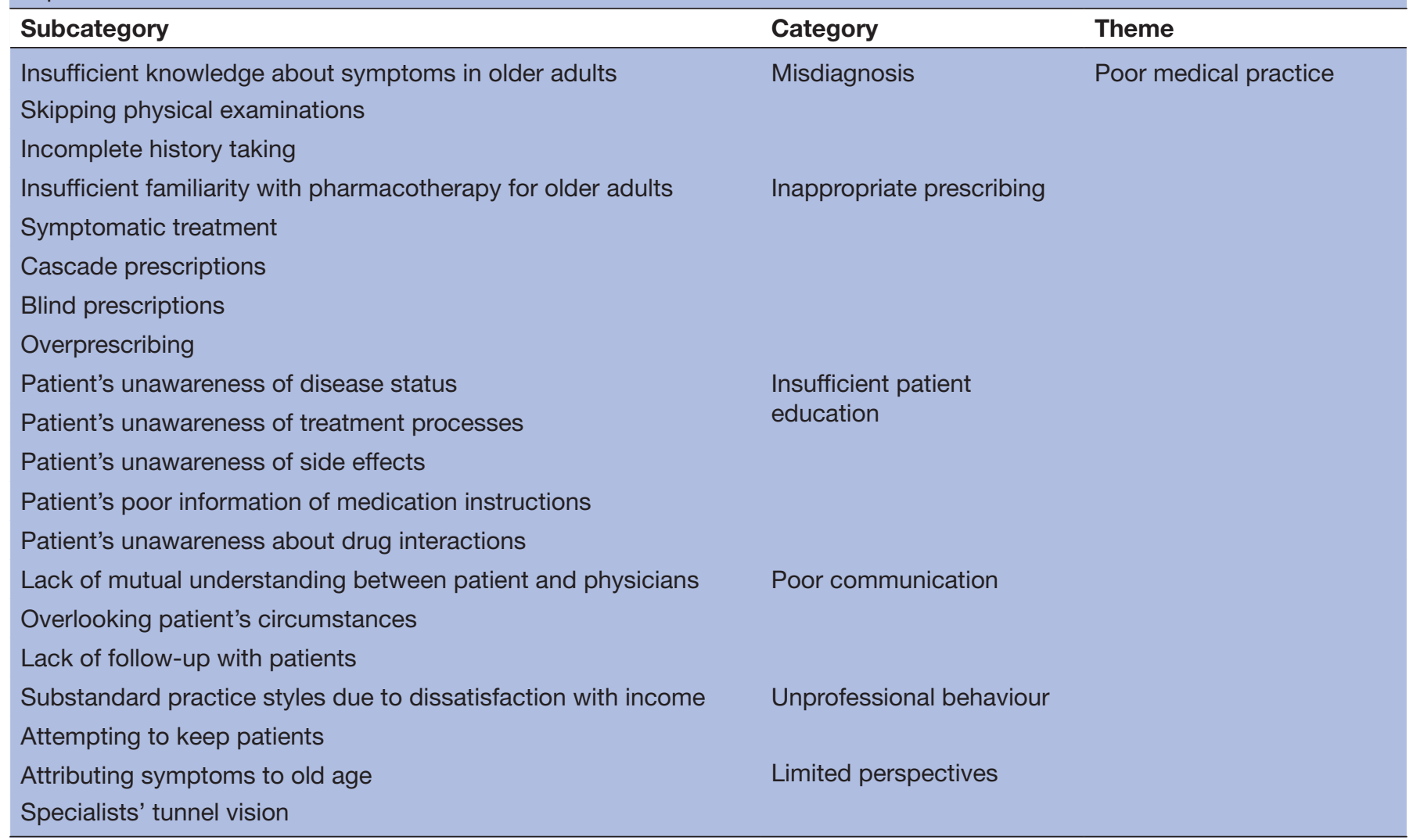

unnecessary or excess medication, but also increases the amount and duration of medication use because the disease is not cured or becomes worse, and this leads to inappropriate polypharmacy.

I had gallbladder pain, but the doctor diagnosed it as gastric pain. I was taking gastric medication for 3 years for nothing. I finally went to another doctor and described the symptoms, and he said it wasn't gastric.(Older adult No. 1)

Inadequate knowledge of symptoms in older patients is a predisposing factor towards physicians' misdiagnosis. 'I had an older adult patient who had depression, but since he'd complained of sleeping problems, they failed to make the right diagnosis and prescribed diazepam. ... Eventually, I visited him in the hospital [admitted with a broken hip] and diagnosed him with depression.' (Physician No. 1)

Participating physicians admitted that, in some cases, thorough history taking and examinations are not possible, especially due to the high demand for services and overcrowded clinics, and as a result, certain errors may occur. 'At public clinics, sometimes I have to see about 60 patients in two hours, and I don't have more than a few minutes for each patient. Many assessments are impossible.' (Physician No. 3)

\section{Inappropriate prescribing}

Based on the described experiences, "inappropriate prescribing' is another issue that makes physicians responsible for inappropriate polypharmacy. Subcategories forming this category were 'insufficient familiarity with therapy for older adults', 'symptomatic treatment', 'cascade prescriptions', 'blind prescriptions' and 'overprescribing'. Our findings indicated that in some cases the diagnosis is correct, but for some reason, such as unawareness about contraindications in older patients, inappropriate medications are prescribed.

I have seen physicians prescribe regular doses of sedatives that should be administered at lower doses for older patients. It's the same with cardiac medications such as digoxin which can be highly toxic. Overall, most of our peers treat older and younger patients the same... (Physician No. 2)

I have seen cases of certain medications with heavy side effects. For instance, a 70-year-old patient was prescribed digoxin, one a day. (Pharmacist No. 3)

In some cases, the physician prescribed medications to treat symptoms which lead to the consumption of multiple medications.

I've seen cases where a physician had prescribed something for each and every symptom. For instance, there was a patient with fever, sore throat, nausea, body aches, and fatigue receiving acetaminophen for fever, metoclopramide for nausea, and injection piroxicam for body pain. It was a simple infectious pharyngitis that needed only one medicine not 7. (Physician No. 6) 
Administration of medication for treating side effects of other medications, or a prescribing cascade, is another issue mentioned in this category.

In one case, the pressure in this 70-year-old lady was 90. She was on hypertensive medication and complained of dizziness at night for which she'd received more medication. The blood pressure shouldn't be so low in older patients. (Physician No. 1)

In some cases, blind prescriptions occur because physicians lack access to patient records, or they begin a course of treatment before running the necessary tests or making a definite diagnosis. Prescribing excessive amounts of medication, particularly antibiotics, is another scenario stated by participating physicians and patients.

You cannot apply the textbook to the patient and ask for a throat culture as the first step. As a result, even when physicians know it might be viral, they prefer to try to play it safe and administer something like amoxicillin. That's why antibiotics are prescribed in most cases. (Physician No. 2)

\section{Insufficient patient education}

Subcategories forming this category were 'patient's unawareness of disease status', 'patient's unawareness of treatment processes', 'patient's unawareness of side effects', 'patient's poor information of medication instructions' and 'patient's unawareness about drug interactions'.

In many cases, inappropriate polypharmacy occurs despite the correct diagnosis and proper prescription of medications by physicians. These cases, according to our data, arise from insufficient patient education by the practitioner. Experiences recounted by patients indicated that physicians do not spend enough time to educate patients, and patients have no clear understanding of what their problems are, what measures are being taken, how long the process takes, what they should do and what they should avoid. In some cases, patients are not even clear about how to use the medications they have received. All patients pointed to the fact that they did not receive sufficient information about the side effects of the prescribed medications, and consequently, they were not aware of what to do in case they experience any problems.

My grandmother consulted a cardiologist and was taking some medications. Then she had a problem and went to the Heart Hospital where several other doctors examined her and prescribed another set of medications without educating her that they were prescribing a new medication for her disease, and that she should discontinue the old one. It was later when I realized she was taking them all together. (Physician No. 7).

'The doctor prescribed the medicines but didn't say anything about what time or how to take them. Besides, they don't tell you what to do if there is a side effect. They don't even tell you that the medication might have side effects. They don't tell you how long to take them, what to do when we run out, what to take them with, or what not to take them with.' (Older adult No.7)

\section{Poor communication}

Poor physician-patient communication is another influential factor in the development of inappropriate polypharmacy among older adults. Subcategories forming 'poor communication' were 'lack of mutual understanding between patient and physicians', 'overlooking patient's circumstances' and 'lack of follow-up with patients'. Overall, the doctor-patient relationship is more complex in case of older patients due to the complexity of their conditions compared with other patients.

Because they won't understand, no matter how much I explain. Besides, they're hard of hearing, and I have to keep shouting. Then if I ask something, they keep talking for onehour, and we don't have time. In the end, even if they understand what I'm saying at the time, they will forget everything as soon as they leave. And since they're illiterate, I have to write everything myself, and it takes too long. (Physician No. 6)

In many cases, the patient does not understand the doctor's orders or misinterprets them. 'The doctor told me to do an exercise test and get an echo, but since I have a treadmill at home, I just used that instead. I told myself I'd exercise on my own at home.' (Older adult No. 1)

Another point expressed by participants was that doctors' orders should be in accordance with patients' overall circumstances, so that it would be applicable and feasible. For example, insurance status, economic status, living conditions (ie, living alone or with family members) are some of the issues that need to be considered. 'I saw a doctor a few months ago, and all he prescribed was import medicines which I can't afford, so I didn't buy them. They're too expensive when you're living on pension. Now my situation is worse, and I'm here to see what can be done.' (Older adult No. 3)

Another complaint raised by most patients was that physicians often have no specific follow-up plan for treatment, and somehow, they are not informed of the next step after they finish the current prescription. 'The majority of physicians that we consulted for mom didn't have a specific treatment plan. Even if they did, they didn't tell us anything about it.' (Caregiver No. 1)

\section{Unprofessional behaviour}

Based on the experiences expressed in this study, physicians' unprofessional behaviour is one of the factors contributing to inappropriate polypharmacy in older adults. Two subcategories of 'substandard practice styles due to dissatisfaction with income' and 'attempting to keep patients' formed this category.

The adverse impact of physicians' discontent with their income on the quality of their services is one of the items outlined in this category. Sometimes, certain measures 
such as patient education are omitted, and this causes patient confusion and error in taking their medications. Sometimes enough time is not allocated to the visit so that more patients can be seen, and this can lead to a misdiagnosis or misprescription. In response to our question about this issue, physicians stated dissatisfaction with income as a reason.

I sense I'm not getting paid enough for the time I spend for each patient, and I'm not happy with my income. That's why I don't spend much time' (Physician No. 6). "Truth is that educating patients takes time, and considering the low visit fees, doctors don't do it. I mean a practitioner can't spend $10-15$ min talking to a patient when the visit fee is so low. That's why they don't. (Physician No. 2)

Another issue raised in this category was trying to win patients' loyalty. Sometimes physicians give in to patients' wishes and demands. For example, they prescribe the medication they ask for, or refer them for diagnostic tests patients wish to do. In some cases, physicians do not refer the patient to specialists or fellows, and they try to prevent losing clients as far as possible.

I have prescribed things they've asked for. ... What option do you have when you see a patient who asks for medicine? If I don't, the next doctor will. I've seen it with other peers as well. For instance, this patient came to me and asked for penicillin because of some old habit from childhood. (Physician No. 3)

\section{Limited perspectives}

One of the issues in this category was that physicians tend to neglect older patients and attribute their symptoms and problems to old age. 'Some physicians say, 'Well, he is 94 years old! What do you expect?' They say it's all because of age, and there's nothing they can do. It might be a side effect of the medications. But they don't care.' (Caregiver No. 2)

Another issue raised in this category is specialists' tunnel vision. This means that practitioners may only look for and treat problems related to their specialty rather than the patient as a whole, and neglect other diseases and medications they are receiving. As far as inappropriate polypharmacy is concerned, this tunnel vision approach can lead to prescribing redundant medications because patients' current medications are not reviewed or administering medications that are contraindicated on account of other concurrent disease.

The problem is that everyone cares about their own field. For a given patient, the cardiologist prescribes heart medicine, the internist prescribes his own, and some other doctor for gastric problems. Eventually, patients end up with a whole bag of medicines. Many of these interact and are not compatible with each other. (Physician No. 2)

I had this patient who had asthma for years, and then developed heart problems. He went to a cardiologist who prescribed propranolol without asking about his diseases... They [physicians] only prescribe their own medications and don't care about patients' other diseases. (Pharmacist No. 1)

\section{DISCUSSION}

Results of this study showed that although physicians can have a preventive role with proper disease management, their poor performance in the treatment process can lead to inappropriate polypharmacy. Misdiagnosis and misprescription by physicians can lead to taking unnecessary and excessive medications by older patients. Influential factors include physicians' insufficient information about geriatric medicine and overlooking their special circumstances. In addition, inappropriate polypharmacy is very likely when older patients are not educated by their physicians about their disease status, treatment process and prescribed medications. The physician-patient relationship and the communication between them is another determinant of this phenomenon. Certain unprofessional behaviours of practitioners lay the groundwork for inappropriate polypharmacy, and in this setting, physicians' performance is affected by their perceptions and attitudes.

\section{Misdiagnosis}

One of the categories that emerged in this study was misdiagnosis. Similarly, as stated in the review article by Chowdhury et al, receiving inappropriate treatment (inappropriate polypharmacy) is one of the consequences of misdiagnosis in epilepsy. ${ }^{31}$ The diagnosis of diseases in older patients is far more complicated, because they have different symptoms compared with other age groups ${ }^{32}$ and lack of physician awareness can hinder the correct diagnosis. This finding is consistent with results of the study by Vegter $e t$ al which found poor physician knowledge as the most important contributor to misdiagnosis. ${ }^{33}$ Given the novelty of geriatric medicine in Iran and absence of a geriatrics course in the general medicine curriculum, poor physician knowledge not unexpected. ${ }^{34}$ Certain measures have been taken to add age-related topics to the curriculum, but they have not been implemented in all medical schools throughout the country. In addition, previous graduates need to receive appropriate training through continued medical education courses. Sarkar suggests that preventing inappropriate polypharmacy in older adults requires interventions to increase knowledge and awareness among physicians; these include discussion of the topics in seminars, conferences, continued education, as well as revising the medical/nursing/pharmacy school curriculum and residency/postgraduate training. ${ }^{7}$

Incomplete history taking and physical examinations are other contributors to misdiagnosis, as expressed by the participants. Medical textbooks consider history taking and physical examinations essential elements for an accurate diagnosis. ${ }^{35}$ In a study by Ikiz et al, the 
authors demonstrated that obtaining a thorough history including present illness, previous chronic disease and family history is an important step in the diagnosis of diseases. ${ }^{36}$ The results of this study suggest that failing to spend enough time to visit the patient leads to incomplete history taking and physical examinations. To provide quality care, it is recommended not to visit more than 3-4 patients per hour. ${ }^{37}$ However, according to the experiences recounted by participating physicians, in some cases it is impossible to adhere to such time allocations, especially in public centres where the number of patients can be overwhelming. As a solution, in some countries such as the USA, certain tasks are undertaken by physician assistants. ${ }^{38}$ In Iran, a physician assistant is not an officially defined entity, and all necessary tasks are done personally by physicians themselves, which requires them to spend more time. One of the main causes of clinic overload, especially in public centres, seems to be the low costs, and further studies are needed to examine possible solutions such as employing trained physician assistants.

\section{Inappropriate prescribing}

Another category that emerged in the present study was inappropriate prescribing which is suggested as one of the major challenges in the treatment of older patients. ${ }^{12-14}$ Exploring the inappropriate medications prescribed to older adults in Iran, Azoulay et al showed that $27.6 \%$ of older patients in Iran have at least one inappropriate medication in their prescriptions. ${ }^{39}$ Based on the participants' experiences, physicians lack sufficient familiarity with medical therapy for older patients and PIM in this age group. This finding is in agreement with the results of a study by Ramaswamy et al. ${ }^{40}$ Although studies indicate that applying tools for evaluating appropriateness of prescribing are an effective way to reduce inappropriate prescribing, currently available tools and guidelines are not well known. ${ }^{9}$ Physicians in Iran have had no education on this subject, and providing training on this subject should be considered.

An important point is that there is no proper guideline for the complex conditions of older patients who may have several chronic comorbidities, and most available guidelines have been prepared for single disease conditions. ${ }^{841}$ Therefore, given the growing population of older age groups, it is essential to design and formulate guidelines for comorbidities.

Another reason for inappropriate prescriptions is lack of access to detailed records of patients' clinical and medical history, and thus, decision-making can only be based on patients' self-reports. Physicians' lack of access to a coherent and secure patient information system is considered an important factor affecting the quality of diagnostic and therapeutic processes in Iran. ${ }^{23}$ Certain measures have been taken to establish electronic medical records for patients, but none of these measures are in place yet.

Interestingly, another topic mentioned by all study participants was the overprescribing of medications by physicians. One of the reasons raised by doctors was the perception that if they do not prescribe medications, patients might interpret it as their inability to make a diagnosis, and they will lose the client. Other studies also show that overprescribing is common among Iranian physicians ${ }^{42}{ }^{43}$; especially antibiotics ${ }^{44}$ and injection medications. ${ }^{42}{ }^{45}$ According to the participants' experiences, there seems to be a misunderstanding between doctors and patients in this regard. On one hand, physicians stated that patients favour using medications and would be unhappy if none is prescribed, and that is why they try to prescribe something even of it would count as placebo. On the other hand, patients refused to see a doctor for fear of having a new item added to their medicine list. It seems necessary to conduct in-depth studies to shed light on different aspects of this issue.

\section{Insufficient patient education}

Patient education is one of the most important pillars of care, and based on available literature, it can play an important role in the prevention of inappropriate polypharmacy. ${ }^{46}$ According to García-Gollarte et al, educational interventions about the use of medications can reduce the use of inappropriate medicines and decrease healthcare service usage among older adults. ${ }^{47}$ Colley and Lucas state that patient education, including them in the decision-making process and explaining treatment goals and plans, can help prevent inappropriate polypharmacy. ${ }^{48}$

However, according to the results of the present study, physicians often overlook this matter. Patients are less likely to adhere to treatment when they are not educated about their treatment measures, the duration of the treatment process, their prescribed medications, the mode of action of the medications and the wait time for the medication to take effect. For example, when they are unaware that it takes a given medication 1 month to take effect, they may consider the medication ineffective and increase the dosage or seek an effective alternative. Participating physicians stated that skipping patient education was mainly due to busy offices and insufficient time, which is commonly seen in public centres. In addition, the subject of patient education lacks a defined process in Iran, and the roles of physicians and pharmacists are not clarified individually. The problem can be partly solved if physicians and pharmacists are given exclusive responsibilities for providing education about treatment processes and medications, respectively, and appropriate legal and monitoring measures are established. Various medications (Over the counter [OTC] and non-OTC items) are easily accessible to patients in Iran without a prescription. This fact highlights the importance of patient education by pharmacists. ${ }^{23} 25$ The role of patient education by pharmacists in improving patient awareness and reducing medication-related events, including polypharmacy, has been discussed and emphasised in Iranian and international studies. ${ }^{49} 50$ 


\section{Poor communication}

The doctor-patient relationship is another influential factor expressed by study participants. In agreement with this finding, Sarkar suggested that a proper doctorpatient relationship can prevent inappropriate polypharmacy. ${ }^{7}$ Older patients have unique circumstances; in addition to low health literacy, poor vision and hearing is more common in older ages and such conditions can impact their ability to communicate. ${ }^{51}$ In addition, multimorbidities are common, and the prevalence rates of memory and cognitive problems are high in older adults. Retirement and economic issues must be added to these problems. $^{358}$

Physicians need to consider all the above factors and establish an effective relationship to select a proper treatment process and educate older patients. In a meta-synthesis regarding the reduction of inappropriate polypharmacy from the point of view of physicians and older patients, Bokhof and Junius-Walker stated that the doctor-patient relationship is essential for logical management of medications, and that good communication and trust between doctors and patients can play a positive role towards reducing inappropriate polypharmacy. ${ }^{52}$ According to Linetzky et al, patient dissatisfaction with their physicians' interaction can reduce their treatment adherence. ${ }^{53}$ Thus, it seems that training for developing doctor-patient communication skills, which is missing from curriculums, is another subject that should be considered for medical programmes.

\section{Unprofessional behaviour}

Unprofessional physician behaviour is another concept that emerged in this study. Based on the results, dissatisfaction with income influenced service delivery in some cases. Other studies conducted in Iran also confirm that physicians' income status is a major source of stress which affects their performance. ${ }^{54} \mathrm{Li}$ et al suggest that income status plays an important role in physician satisfaction with their occupation. ${ }^{55}$ According to Rothenberg et $a l$, physicians' performance is influenced by financial incentives including base salary, overtime and agreement contracts. $^{56}$

Physicians' dissatisfaction with their income can be followed by making efforts to maintain clients in their practice. Some patients may be consulting the wrong specialist due to lack of awareness or proper referral. Such issues can be associated with misdiagnosis and consequently prescribing incorrect or unnecessary medication. In some cases, it can lead to complications or exacerbation of the disease, and prolonged complicated treatment, because proper interventions have not been implemented by the right person at the right time.

Although, the system sets the stage for this problem; since there is no referral system in the specialty and subspecialty level, physicians are not sure if referred patients will return to their practice, and thus, they try not to refer them as far as possible. Casalino and Crosson also state that doctors' dissatisfaction can lead to a variety of consequences including diminished physician concentration, effort, empathy and professionalism. ${ }^{57}$

\section{Limited perspectives}

Many of the study participants, particularly those in the older age groups, recalled having received less attention and having their conditions and symptoms attributed to their age. Other studies confirm this finding. ${ }^{58}$ In addition, many patients themselves attribute their symptoms to their old age, ${ }^{59}$ and this can lead to the aggravation of patients' symptoms and conditions. According to Ben-Harush et al, physicians can be less inclined to refer older patients for diagnostic procedures and less willing to intervene compared with younger patients. ${ }^{58}$ Such attitudes can be interpreted as ageism which was somehow reflected in other categories as well. For example, some physicians seemed to prefer not to deal with elderly patients and failed to communicate with and educate them properly. Another example, presented under the inappropriate prescribing category, was treating older patients with the same prescription given to younger adults without respecting age-related differences and adjusting for the dose or form of medications. According to Fialová et al, even such non-discriminating 'age-blind' approaches can be interpreted as ageism as well. ${ }^{60}$

Some experiences of limited perspectives were in relation to specialists and subspecialists. While specialists and subspecialists can play an important role in enhancing patient care, challenges such as operating in silos and lack of coordinated care can take a toll on the quality and cost of healthcare. In the current healthcare system in Iran, such lack of integration can contribute to fragmentation of care, threaten the integrity of healthcare services, and have undesirable outcomes, especially for elderly patients who are more likely to have multimorbidities. ${ }^{61} 62$

In Iran, the referral system, especially in urban and suburban settings, is one of the challenges of the health system. As such, the patient can freely consult any doctor and specialist they wish. ${ }^{24}$ However, if practitioners focus only on the main complaint of the patient and overlook other concurrent problems, prescriptions may include medications that are similar, from the same class, or even contraindicated for a given patient. This matter can become problematic because there is no coordination among various service providers to share diagnostic and therapeutic information, and there is no system in place to monitor medications prescribed by different doctors and prevent potential issues. Holmes et al suggest that addressing inappropriate prescribing requires reduced fragmentation of care, because a significant relationship was observed between a higher number of prescribers and use of PIM. ${ }^{63}$ Castro and Billick discuss how physicians develop tunnel vision during their residency and fellowship training. Physicians with tunnel vision are likely to overlook other possible causes of symptoms, which are beyond their specialty area. In addition to mastering their own specialty field, specialists must consider other possible causes of disease. ${ }^{64}$ 


\section{Limitations}

This study was conducted in the context of the Iranian culture and health system, and differences should be taken into consideration when interpreting results. In addition, due to the sensitivity of the study subject, it is likely that certain experiences were not discussed. Given the negative impact of physician error, physicians' responses may have been influenced by social desirability bias, and certain experiences may not have been reported. This limitation was addressed during interviews with non-physician participants to provide comprehensive data.

Identifying patients with lived experience of inappropriate polypharmacy requires clinical and paraclinical evaluations as well as a specialty review of their list of medications. Given our limited resources and the increased risk of inappropriate polypharmacy with the use of a higher number of medications, the initial inclusion criterion was in accordance with the count-based definition of polypharmacy (use of four medications or more). For those who met this criterion, the MAI was administered by a physician to select participants with inappropriate polypharmacy.

\section{CONCLUSION}

Inappropriate polypharmacy is an interactive and complex phenomenon influenced by various factors, and its development varies in different populations. This study focused on the role of physicians in the development of polypharmacy among Iranian older adults. Based on the results of this study, the performance, knowledge, vision and attitudes of physicians may, directly and indirectly, lead to inappropriate polypharmacy. In addition, as suggested by our findings, multiple other elements seem to contribute to the physicians' role in the development or prevention of this phenomenon. These include physicians' practice settings and the policies and regulations that govern medical practices in the national healthcare system (eg, the referral system, especially at levels higher than primary and general physician care); tackling the challenge of polypharmacy requires such issues to be addressed as well. Further research using grounded theory is recommended to explore the role of patients, pharmacists and the system which defines and provides healthcare services. This can help identify other concepts and categories, better understand the relationship between concepts, generate theories, and reach a deeper understanding of the collection of factors that contribute to polypharmacy in the setting of the Iranian healthcare system.

Acknowledgements The authors wish to express their gratitude to all study participants who shared their experience. The authors also thank Mehrdad Eftekhar, Seyed Vahid Shariat and Mojgan Taban (Mental Health Research Centre of Iran University of Medical Sciences) for administrative support.

Contributors SSM, MS and SKM contributed to design the study, conceptualisation, data collection, formal analysis, methodology, writing original draft, review and editing. HRK and FA: contributed to supervision, visualisation, methodology, validation, writing original draft, review and editing. ShM contributed to investigation, project administration, supervision, methodology, writing the original draft and review and editing of the manuscript.

Funding This work was financially sponsored by Iran University of Medical Sciences as a PhD studentship (grant no: 93-121-02-24651).

Competing interests None declared.

Patient consent for publication Not required.

Ethics approval This study was approved as a minimal-risk research by the Ethics Committee of Iran University of Medical Sciences (approval reference number: IR.IUMS.REC.1393-02-121-24651).

Provenance and peer review Not commissioned; externally peer reviewed.

Data sharing statement The data that support the findings of this study are available on request from the first author, not including personal identifiers based on our informed consent.

Open access This is an open access article distributed in accordance with the Creative Commons Attribution Non Commercial (CC BY-NC 4.0) license, which permits others to distribute, remix, adapt, build upon this work non-commercially, and license their derivative works on different terms, provided the original work is properly cited, appropriate credit is given, any changes made indicated, and the use is non-commercial. See: http://creativecommons.org/licenses/by-nc/4.0/.

\section{REFERENCES}

1. Acurcio FA, Perini E, Magalhães SMS, et al. Analysis of medical prescriptions dispensed at health centers in Belo Horizonte, Minas Gerais, Brazil. Cadernos de Saúde Pública 2004;20:72-9.

2. Elmståhl S, Linder H. Polypharmacy and Inappropriate Drug Use among Older People-a Systematic Review. Healthy Aging \& Clinical Care in the Elderly 2013;5:1.

3. Fulton MM, Allen ER. Polypharmacy in the elderly: a literature review. J Am Acad Nurse Pract 2005;17:123-32.

4. Hilmer SN, Gnjidic D. The effects of polypharmacy in older adults. Clin Pharmacol Ther 2009;85:86-8.

5. Patterson SM, Hughes $\mathrm{C}$, Kerse N, et al. Interventions to improve the appropriate use of polypharmacy for older people: The Cochrane Library, 2012.

6. Bushardt RL, Massey EB, Simpson TW, et al. Polypharmacy: misleading, but manageable. Clin Interv Aging 2008;3:383-9.

7. Sarkar S. Geriatric Poly-Pharmacy: A Growing Epidemic. How to Prevent It? Br J Med Med Res 2017;21:1-11.

8. Mortazavi SS, Shati M, Keshtkar A, et al. Defining polypharmacy in the elderly: a systematic review protocol. BMJ Open 2016;6:e010989.

9. O'Connor MN, Gallagher P, O'Mahony D. Inappropriate Prescribing. Drugs Aging 2012;29:437-52.

10. Steinman MA, Landefeld CS, Rosenthal GE, et al. Polypharmacy and prescribing quality in older people. J Am Geriatr Soc 2006;54:1516-23.

11. Hill-Taylor B, Sketris I, Hayden J, et al. Application of the STOPP/START criteria: a systematic review of the prevalence of potentially inappropriate prescribing in older adults, and evidence of clinical, humanistic and economic impact. J Clin Pharm Ther 2013;38:360-72.

12. Bregnhøj L, Thirstrup S, Kristensen MB, et al. Reliability of a modified medication appropriateness index in primary care. Eur J Clin Pharmacol 2005;61:769-73.

13. Fick DM, Cooper JW, Wade WE, et al. Updating the Beers criteria for potentially inappropriate medication use in older adults: results of a US consensus panel of experts. Arch Intern Med 2003;163:2716-24.

14. O'Mahony D, O'Sullivan D, Byrne S, et al. STOPP/START criteria for potentially inappropriate prescribing in older people: version 2. Age Ageing 2015;44:213-8.

15. Gnjidic D, Hilmer SN, Blyth FM, et al. Polypharmacy cutoff and outcomes: five or more medicines were used to identify communitydwelling older men at risk of different adverse outcomes. J Clin Epidemiol 2012;65:989-95.

16. Hayes BD, Klein-Schwartz W, Barrueto F. Polypharmacy and the geriatric patient. Clin Geriatr Med 2007;23:371-90.

17. Rambhade S, Chakarborty A, Shrivastava A, et al. A survey on polypharmacy and use of inappropriate medications. Toxicol Int 2012;19:68.

18. Iran Statistical Center. Report of the 2011 census of housing and population of Iran. Tehran, Iran: Iran Statistical Center, 2013. 
19. Taheri Tanjani P, Moradinazar M, Esmail Mottlagh M, et al. The prevalence of diabetes mellitus (DM) type II among Iranian elderly population and its association with other age-related diseases, 2012. Arch Gerontol Geriatr 2015;60:373-9.

20. Tanjani PT, Motlagh ME, Nazar MM, et al. The health status of the elderly population of Iran in 2012. Arch Gerontol Geriatr 2015;60:281-7.

21. Dianati M, Shojaegharebag G, Mesdaghinia A, et al. Polypharmacy and its related factors among the elderly population in Kashan, Iran during 2011-2012. Feyz Journal of Kashan University of Medical Sciences 2015;18:578-84.

22. Sharifi H, Hasanloei MA, Mahmoudi J. Polypharmacy-induced drugdrug interactions; threats to patient safety. Drug Res 2014;64:633-7.

23. Mehrdad R. Health system in Iran. JMAJ 2009;52:69-73.

24. Moradi-Lakeh M, Vosoogh-Moghaddam A. Health Sector Evolution Plan in Iran; Equity and Sustainability Concerns. Int $J$ Health Policy Manag 2015;4:637-40.

25. Mortazavi SS, Shati M, Khankeh HR, et al. Self-medication among the elderly in Iran: a content analysis study. BMC Geriatr 2017; $17: 198$.

26. Graneheim UH, Lundman B. Qualitative content analysis in nursing research: concepts, procedures and measures to achieve trustworthiness. Nurse Educ Today 2004:24:105-12.

27. World Health Organization. Ageing and health. 2015 http://www.who. $\mathrm{int} / \mathrm{mediacentre/factsheets/fs404/en/} \mathrm{(Accessed} 9$ Jan 2017).

28. Elo S, Kyngäs $\mathrm{H}$. The qualitative content analysis process. J Adv Nurs 2008;62:107-15.

29. Schwandt TA, Lincoln YS, Guba EG. Judging interpretations: But is it rigorous? trustworthiness and authenticity in naturalistic evaluation. New Dir Eval 2007;2007:11-25.

30. Tong A, Sainsbury P, Craig J. Consolidated criteria for reporting qualitative research (COREQ): a 32-item checklist for interviews and focus groups. Int J Qual Health Care 2007;19:349-57.

31. Chowdhury FA, Nashef L, Elwes RD. Misdiagnosis in epilepsy: a review and recognition of diagnostic uncertainty. Eur $J$ Neurol 2008; 15:1034-42.

32. Fiske A, Wetherell JL, Gatz M. Depression in older adults. Annu Rev Clin Psychol 2009;5:363-89.

33. Vegter S, de Jong-van den Berg LT. Misdiagnosis and mistreatment of a common side-effect--angiotensin-converting enzyme inhibitorinduced cough. Br J Clin Pharmacol 2010;69:200-3.

34. Fereshteh A, Tabibi SJ, Maleki MR, et al. A prediction of the geriatric specialists for the elderly in Iran: a dynamic system. WALIA journal 2015;31(S2):132-8.

35. Bickley L, Szilagyi PG. Bates' guide to physical examination and history-taking. 11 th edn: Lippincott Williams \& Wilkins, 2012.

36. Ikiz MA, Cetin II, Ekici F, et al. Pediatric syncope: is detailed medical history the key point for differential diagnosis? Pediatr Emerg Care 2014;30:331-4.

37. Dugdale DC, Epstein R, Pantilat SZ. Time and the patient-physician relationship. J Gen Intern Med 1999;14(S1):S34-40.

38. Hutchinson L, Marks T, Pittilo M. The physician assistant: would the US model meet the needs of the NHS? BMJ 2001;323:1244-7.

39. Azoulay L, Zargarzadeh A, Salahshouri Z, et al. Inappropriate medication prescribing in community-dwelling elderly people living in Iran. Eur J Clin Pharmacol 2005;61:913-9.

40. Ramaswamy R, Maio V, Diamond JJ, et al. Potentially inappropriate prescribing in elderly: assessing doctor knowledge, confidence and barriers. J Eval Clin Pract 2011;17:1153-9.

41. Stefanacci RG, Khan T. Can Managed Care Manage Polypharmacy? Clin Geriatr Med 2017;33:241-55.

42. Safaeian L, Mahdanian AR, Hashemi-Fesharaki M, et al. General physicians and prescribing pattern in isfahan, iran. Oman Med $J$ 2011;26:205-6.

43. Soleymani F, Taheri F. Evaluation of Medicine Prescription Pattern Using World Health Organization Prescribing Indicators in Iran: A Cross-Sectional Study. Value Health 2014;17:A447.
44. Safaeian L, Mahdanian AR, Salami S, et al. Seasonality and Physician-related Factors Associated with Antibiotic Prescribing: A Cross-sectional Study in Isfahan, Iran. Int J Prev Med 2015;6:1.

45. Cheraghali AM, Solemani F, Behmanesh Y, et al. Physicians' Attitude Toward Injectable Medicines. Journal of Pharmacology and Toxicology 2006;1:33-9.

46. Tannenbaum C, Martin P, Tamblyn R, et al. Reduction of inappropriate benzodiazepine prescriptions among older adults through direct patient education: the EMPOWER cluster randomized trial. JAMA Intern Med 2014;174:890-8.

47. García-Gollarte F, Baleriola-Júlvez J, Ferrero-López I, et al. An educational intervention on drug use in nursing homes improves health outcomes resource utilization and reduces inappropriate drug prescription. J Am Med Dir Assoc 2014;15:885-91.

48. Colley CA, Lucas LM. Polypharmacy. J Gen Intern Med 1993;8:278-83.

49. Garjani A, Rahbar M, Ghafourian T, et al. Relationship of pharmacist interaction with patient knowledge of dispensed drugs and patient satisfaction. East Mediterr Health J 2009;15:934-43.

50. Maher RL, Hanlon J, Hajjar ER. Clinical consequences of polypharmacy in elderly. Expert Opin Drug Saf 2014;13:57-65.

51. Cohen JM, Blustein J, Weinstein BE, et al. Studies of PhysicianPatient Communication with Older Patients: How Often is Hearing Loss Considered? A Systematic Literature Review. J Am Geriatr Soc 2017;65:1642-9.

52. Bokhof B, Junius-Walker U. Reducing Polypharmacy from the Perspectives of General Practitioners and Older Patients: A Synthesis of Qualitative Studies. Drugs Aging 2016;33:249-66.

53. Linetzky B, Jiang D, Funnell MM, et al. Exploring the role of the patient-physician relationship on insulin adherence and clinical outcomes in type 2 diabetes: Insights from the MOSAlc study. $J$ Diabetes 2017;9:596-605.

54. Tavakoli Z, Montazeri A, Farshad AA, et al. Sources of Stress and Coping Strategies among Iranian Physicians. Glob J Health Sci 2016;9:120.

55. Li T, Lei T, Sun F, et al. Determinants of village doctors' job satisfaction under China's health sector reform: a cross-sectional mixed methods study. Int J Equity Health 2017;16:64.

56. Rothenberg R, Koplan JP, Cutler C, et al. Changing pediatric practice in a changing medical environment: factors that influence what physicians do. Pediatr Ann 1998;27:241-50.

57. Casalino LP, Crosson FJ, Satisfaction P. and Physician WellBeing: Should Anyone Care? Professions and Professionalism 2015;5:1-12.

58. Ben-Harush A, Shiovitz-Ezra S, Doron I, et al. Ageism among physicians, nurses, and social workers: findings from a qualitative study. Eur J Ageing 2017;14:39-48.

59. Stewart TL, Chipperfield JG, Perry RP, et al. Attributing illness to 'old age:' consequences of a self-directed stereotype for health and mortality. Psychol Health 2012;27:881-97.

60. Fialová D, Kummer I, Držaić M, et al. Ageism in medication use in older patients. Contemporary perspectives on ageism: Springer, 2018:213-40.

61. Detsky AS, Gauthier SR, Fuchs VR. Specialization in medicine: how much is appropriate? JAMA 2012;307:463-4.

62. Ehrich JH, Kerbl R, Pettoello-Mantovani M, et al. Opening the Debate on Pediatric Subspecialties and Specialist Centers: Opportunities for Better Care or Risks of Care Fragmentation? J Pediatr 2015;167:1177-8.

63. Holmes HM, Luo R, Kuo YF, et al. Association of potentially inappropriate medication use with patient and prescriber characteristics in Medicare Part D. Pharmacoepidemiol Drug Saf 2013;22:728-34.

64. Castro J, Billick S. Psychiatric presentations/manifestations of medical illnesses. Psychiatr Q 2013;84:351-62. 